\title{
Alterações músculo- esqueléticas do envelhecimento, prevenção e atuação fisioterapêutica nas quedas em idosos: revisão bibliográfica
}

\author{
Musculoskeletal changes in aging, prevention and physiotherapy in falls in the elderly: a \\ bibliographic review
}

Cambios musculoesqueléticos em el envejecimiento, prevención y fisioterapia em caídas em el anciano: uma revisión bibliográfica

Pedro Rafael Dourado Dos Santos ORCID: https://orcid.org/0000-0002-9230-142X Instituto Educacional Santa Catarina, Brasil E-mail: p.d.rafael@hotmail.com

Rayssa Rafaela Dourado Dos Santos ORCID: https://orcid.org/0000-0003-2559-6229 Instituto Educacional Santa Catarina, Brasil E-mail: rayssarafaeladouradodossantos@gmail.com

Karla Camila Correia da Silva

ORCID: https://orcid.org/0000-0003-1538-7028 Instituto Educacional Santa Catarina, Brasil Faculdade Guaraí, Brasil

E-mail: karlacamilac@yahoo.com.br

Lécia Kristine Lourenço

ORCID: https://orcid.org/0000-0002-0531-7221 Instituto Educacional Santa Catarina, Brasil Faculdade Guaraí, Brasil E-mail: leciakristine@yahoo.com.br

\begin{abstract}
Resumo
O envelhecimento é um processo dinâmico, progressivo e irreversível e é ligado intimamente com fatores biológicos, psíquicos e sociais, o sistema musculoesquelético e ósseo é crucial no processo do envelhecimento, a queda é a principal causa externa de morbidade e mortalidade entre essa população no mundo e é também um indicador de piora na qualidade de vida, a Fisioterapia entra com um papel importantíssimo tanto na prevenção de quedas, como na reabilitação desses indivíduos, utilizando diversas técnicas. O objetivo deste trabalho foi aprofundar e analisar sobre as alterações musculoesqueléticas do envelhecimento, prevenção e atuação fisioterapêutica nas quedas em idosos. Pesquisa bibliográfica, descritiva. Um dos principais sinais do envelhecimento é a menor capacidade para atividades laborais do trabalho, a adaptação ao ambiente e independência funcional. A Fisioterapia entra com um papel importantíssimo tanto na prevenção de quedas, como na reabilitação desses indivíduos, utilizando de técnicas como, alongamentos, fortalecimento muscular, treino de marcha e equilíbrio, melhora da propriocepção, buscando a manutenção ou melhoria da capacidade funcional, redução das incapacidades e limitações e proporcionando maior independência funcional. Esse estudo concluiu que com o índice de envelhecimento crescente e o aumento das quedas na população idosa esse estudo se tornou de grande relevância, pois esse evento pode provocar graves consequências, diminuindo a autonomia, causando dependência funcional e diminuição na qualidade de vida e a Fisioterapia entra como forte propiciadora da melhor qualidade de vida dessa população.
\end{abstract}

Palavras-chave: Acidentes por queda; Idosos; Fisioterapia.

\begin{abstract}
Aging is a dynamic, progressive and irreversible process and is closely linked to biological, psychological and social factors, the musculoskeletal and bone system is crucial in the aging process, falling is the main external cause of morbidity and mortality among this population worldwide and it is also an indicator of worsening quality of life, Physiotherapy has a very important role both in the prevention of falls and in the rehabilitation of these individuals, using different techniques. The objective of this work was to deepen and analyze the musculoskeletal changes of aging, prevention and physiotherapeutic performance in falls in the elderly. Bibliographic, descriptive research. One of the main signs of aging is the reduced capacity for work activities at work, adaptation to the environment and functional independence. Physiotherapy has a very important role both in the prevention of falls and in the rehabilitation of these individuals, using techniques such as stretching, muscle strengthening, gait and balance
\end{abstract}


training, improvement of proprioception, seeking to maintain or improve functional capacity, reducing disabilities and limitations and providing greater functional independence. This study concluded that with the increasing aging rate and the increase in falls in the elderly population, this study has become of great relevance, as this event can cause serious consequences, reducing autonomy, causing functional dependence and decreased quality of life and Physiotherapy it enters as a strong provider of the best quality of life for this population.

Keywords: Fall accidents; Elderly; Physiotherapy.

\begin{abstract}
Resumen
El envejecimiento es un proceso dinámico, progresivo e irreversible y está íntimamente ligado a factores biológicos, psicológicos y sociales, el sistema musculoesquelético y óseo es crucial en el proceso de envejecimiento, la caída es la principal causa externa de morbilidad y mortalidad entre esta población a nivel mundial y es También un indicador de empeoramiento de la calidad de vida, la fisioterapia tiene un papel muy importante tanto en la prevención de caídas como en la rehabilitación de estos individuos, utilizando diferentes técnicas. El objetivo de este trabajo fue profundizar y analizar los cambios musculoesqueléticos del envejecimiento, prevención y desempeño fisioterapéutico en caídas en ancianos. Investigación descriptiva y bibliográfica. Uno de los principales signos del envejecimiento es la reducida capacidad para las actividades laborales en el trabajo, la adaptación al entorno y la independencia funcional. La fisioterapia tiene un papel muy importante tanto en la prevención de caídas como en la rehabilitación de estos individuos, utilizando técnicas como estiramientos, fortalecimiento muscular, entrenamiento de la marcha y el equilibrio, mejora de la propiocepción, buscando mantener o mejorar la capacidad funcional, reduciendo discapacidades y limitaciones. y proporcionando una mayor independencia funcional. Este estudio concluyó que con el aumento de la tasa de envejecimiento y el aumento de caídas en la población anciana, este estudio se ha vuelto de gran relevancia, ya que este evento puede ocasionar graves consecuencias, disminuyendo la autonomía, provocando dependencia funcional y disminución de la calidad de vida y fisioterapia en la que ingresa. como un fuerte proveedor de la mejor calidad de vida para esta población.
\end{abstract}

Palabras clave: Accidentes de caída; Anciani; Fsioterapia.

\title{
1. Introdução
}

A população envelheceu e isso se tornou uma realidade tanto no Brasil como no mundo, o Brasil ocupava em 1960 o $16^{\circ}$ lugar e será a $6^{\circ}$ em 2025 e faixa etária de oitenta anos será a de maior crescimento (Gomes et al, 2016).

Aproximadamente $15 \%$ dos brasileiros, 32 milhões de pessoas em 2025 ocuparão o $6^{\circ}$ lugar no número de crescimento do envelhecimento no Brasil, isso irá acontecer por conta da diminuição de taxa de fecundidade e aumento da expectativa de vida, o que gera maior demanda de serviços de saúde por essa população (Oliveira et al, 2017).

Claramente o envelhecimento vai variar de indivíduo para indivíduo, pois é gradativo e para alguns ocorrem mais rapidamente, dependendo de diversos fatores como o estilo de vida, as condições socioeconômicas e doenças crônicas (Vieira et al, 2019).

O envelhecimento biológico acontece no plano molecular, celular, tissular e orgânico, outro envelhecimento é o psíquico que é relacionado com as dimensões cognitivas e psicoafetivas, falar de envelhecimento é abrir um leque de interpretações (Fechine \& Trompieri, 2012).

O ser humano sempre se preocupou com o envelhecimento e existe uma dimensão heterogênica, alguns vão caracterizar o envelhecimento pela diminuição geral das capacidades de vida diária, outros pelo crescente período de vulnerabilidade, outros ainda definem a velhice como o ponto mais alto da sabedoria e cada uma dessas condições correspondem a uma verdade parcial (Araújo et al, 2014).

O envelhecimento é um processo dinâmico, progressivo e irreversível e é ligado intimamente com fatores biológicos, psíquicos e sociais (Fechine \& Trompieri, 2012).

O sistema musculoesquelético e ósseo é crucial no processo do envelhecimento, os músculos esqueléticos são a maior massa de tecido do corpo humano, com cerca de $50 \%$ do peso corporal e é de extrema importância para homeostasia bioenergética em repouso ou em exercício, é o principal local de transformação e armazenamento de energia, é o primeiro suporte dos sistemas cardiovascular e pulmonar (Esquenazi et al, 2014).

A hipertrofia pode levar ao aumento das fibras e a atrofia pelo desuso pode levar as fibras a ficar delgadas, a 
multiplicidade de movimentos que o indivíduo é capaz de realizar está envolvido com a natureza plástica do tecido muscular, o mesmo músculo ou grupo, pode adaptar-se a um movimento (Oliveira et al, 2017).

As alterações fisiológicas do envelhecimento podem levar a uma atrofia e fraqueza da musculatura como, por exemplo, a sarcopenia, porém o seu grau vai depender das alterações endócrinas, nutricionais, mitocondriais, genéticas e comportamentais, como o sedentarismo (Esquenazi et al, 2014).

A perda óssea acontece em indivíduos com mais de setenta anos e atinge o osso cortical e trabecular o que vai caracterizar a osteoporose grau II ou senil (Vieira et al, 2019).

O osso é fundamental porque a sua função é sustentar o corpo é um tecido ativo, com o envelhecimento ficam fragilizados e esses ossos frágeis apoiados à musculatura também fraca levam a quedas que é a consequência mais temida na velhice (Esquenazi et al, 2014).

A queda senil é definida como uma síndrome geriátrica, um evento sem intenção e acaba por resultar em uma mudança severa na vida do indivíduo idoso (Gomes et al, 2016).

A prevalência de quedas em idosos e resulta em hospitalizações, enormes gastos públicos com saúde e o uso dos leitos hospitalares por mais tempo (Teixeira et al, 2019).

A queda é a principal causa externa de morbidade e mortalidade entre essa população no mundo e é também um indicador de piora na qualidade de vida (Leitão et al, 2018).

É um problema de saúde pública, a Fisioterapia atua nessa área, em longo prazo as metas terapêuticas é a prevenção das fraturas e deformidades, as estratégias de tratamento fisioterápico consistem em três etapas que são a prevenção de fatores de risco que predispõe essas quedas, evitar a progressão de patologias já instaladas e promover a profilaxia de quedas e fraturas (Teixeira et al, 2019).

O campo da atuação da Fisioterapia vem crescendo, além do enfoque na reabilitação ela atua também na prevenção e promoção da saúde (Oliveira et al, 2017).

Diante dessa temática esse estudo teve como objetivo, aprofundar e analisar sobre as alterações músculo- esqueléticas do envelhecimento, prevenção e atuação fisioterapêutica nas quedas em idosos.

No mesmo período do iluminismo, por volta de meados do século XIX, houve o surgimento de uma corrente de pensamento filosófico denominada de positivista. Esta se baseava no princípio de que o conhecimento científico devia ser reconhecido como o único conhecimento verdadeiro e absoluto, o mesmo não poderia ser contestado (Galvão et al, 2016).

O maior ícone do positivismo foi Auguste Comte (1798-1895), ele formulou três fases da evolução do pensamento humano: o teológico, o metafísico e o positivo. Contudo, considerava o último como importante para o desenvolvimento da sociedade (Mesquida, 2001). Vários autores consideram que Comte foi o pioneiro nas correntes positivistas, mas de acordo com Iskandar e Leal (2002), ele foi influenciado por vários outros pensadores, como por exemplo, Turgot, Condercet e SaintSimon.

A partir do positivismo de Comte a Educação Científica passa a ser essencial na Educação geral, pois compreendese que através dela a ordem social se estabelece (Souza, 2020). Todavia, para que a Educação Científica seja empregada nos níveis de educação de forma precisa, torna-se fundamental que a Ciência não seja compreendida como uma verdade absoluta, mas um conhecimento que está em constante processo de descobertas e mudanças.

A Educação Científica tem sido alvo de vários debates, sobretudo no modo como ela ocorre no ensino básico público de nosso país. De acordo com Roitman (2007), a educação científica, é ministrada apenas no ensino médio por meio de disciplinas tais como Biologia, Química e Física. Entretanto, esse ensino é de baixa qualidade por diversos fatores, dentre eles está à má formação docente, ausência de laboratórios, material pedagógico desatualizado, carga horária exaustiva e o baixo salário. 
De acordo com Galvão et al. (2016) a ciência apresenta muitos traços de correntes filosóficas que existiram na história da humanidade. Exemplo disso é o pensamento positivista que impera nos mais variados níveis de ensino da Educação Básica brasileira. Por não haver uma preocupação por parte das coordenadorias regionais de educação quanto às manifestações dessas correntes nas escolas de ensino básico, com este trabalho objetivou-se evidenciar a manifestação positivista no que concerne à educação científica em uma escola de ensino fundamental da cidade de Quixelô - Ceará, contribuindo assim, para um maior entendimento do que leva a permanência do positivismo na educação.

\section{Metodologia}

Para alcançar os objetivos será realizada uma pesquisa bibliográfica, com o propósito de auxiliar e compartilhar conhecimentos acerca das alterações músculo- esqueléticas do envelhecimento, prevenção e atuação fisioterapêutica nas quedas em idosos.

A pesquisa bibliográfica por meio de referências teóricas, que podem ter sido publicadas em livros, artigos, dissertações e até mesmo teses, busca a explicação de um problema, procurando também conhecer e analisar artigos e publicações acerca de um determinado tema, problema ou assunto (Bervian \& Silva, 2006).

$\mathrm{O}$ trabalho é de natureza qualitativa, a pesquisa qualitativa compreende um conjunto de diferentes técnicas interpretativas que visam a descrever e a decodificar os componentes de um sistema complexo de significados. Tem por objetivo traduzir e expressar o sentido dos fenômenos do mundo social; trata-se de reduzir a distância entre indicador e indicado, entre teoria e dados, entre contexto e ação (Maanen, 1979A, P.520).

A pesquisa é fundamentada no referencial bibliográfico, a literatura científica utilizada tem origem no idioma da língua portuguesa, inglesa e espanhola, tendo sido considerado, neste estudo, artigos e livros, os trabalhos utilizados serão aqueles publicados nos últimos dez anos.

Os artigos foram selecionados a partir das seguintes palavras-chave indexadas nos Descritores em Ciências da Saúde (DeCS): "accidental falls" (“acidentes por quedas"), "elderly" ("idoso") e "Physiotherapy" ("Fisioterapia"), sendo utilizadas todas as combinações possíveis, com os recursos disponibilizados pelos operadores boolianos AND e OR. Um programa gerenciador de referências (EndNote X7 para Windows \& Thomson Reuters 2013)

Artigos originais nas línguas inglesa e portuguesa foram considerados aptos para inclusão na revisão, os critérios de inclusão foram artigos que o tivessem como objetivo a fisioterapia no tratamento e prevenção das quedas em idosos; avaliar quedas em idosos no Brasil; conter em seu texto informações quantitativas sobre prevalência ou incidência de quedas os fatores de risco e/ou suas circunstâncias e de exclusão estudos repetitivos, e que não se enquadraram nos critérios de inclusão.

Para que o trabalho apresente consistência teórica, serão utilizadas as Plataformas de Pesquisa Bireme, a Base de Dados Literatura Latino-Americana e do Caribe em Ciências da Saúde (LILACS), PUBMED, CAPES e nas bases de dados Scientific Electronic Library Online (SciELO).

\section{Resultados e discussão}

\subsection{Alterações músculo-esqueléticas no envelhecimento}

As alterações fisiológicas intrínsecas do envelhecimento são sutis e são inaptas a gerar qualquer incapacidade na fase inicial e com o passar dos anos causa crescentes níveis de limitações em todos os sistemas corporais que são fundamentais para a manutenção do equilíbrio corporal (Esquenazi et al, 2014).

Um exemplo é o processamento visual que está inteiramente ligado ao movimento e á relações espaciais, o seu enfraquecimento predispõe a quedas (Araújo et al, 2014).

Segundo Teixeira; Andrade; Santos; Caires (2019) o envelhecimento musculoesquelético se torna bem evidente com o 
passar da idade, como por exemplo, a diminuição do comprimento, elasticidade e número de fibras, notável também a perda da massa muscular e elasticidade dos tendões e ligamentos, a uma perda também na viscosidade dos fluidos sinoviais.

Em um estudo de Janssen et al. (2000) eles detectaram com a ressonância magnética e a tomografia computadorizada em 468 indivíduos entre 18 e 98 anos e perceberam que ocorria um declínio de massa muscular que se iniciava na quinta década de vida e um declínio também de 1,9Kg para homens e 1,1 Kg em mulheres.

Para Teixeira; Andrade; Santos; Caires (2019) essa perda ocorre por conta das alterações relacionadas ao envelhecimento e destaca a diminuição da densidade óssea a menor sensibilidade à insulina, menor capacidade aeróbia, menor taxa de metabolismo basal, menor força muscular, menores níveis de atividades físicas diárias.

Depois dos 30 anos ocorre à redução na secção transversal do músculo, a atrofia é detectada gradativamente pelas perdas seletivas das fibras esqueléticas, outra informação pertinente é sobre o ganho de gordura, substituindo a perda da massa muscular que é comum na população senil, o que ajuda a um possível aparecimento de doenças crônicas e incapacidades (Oliveira et al, 2017).

Provavelmente a perda desse tecido muscular resulta na diminuição da força muscular, o pico máximo de força muscular acontece por volta dos 25 a 30 anos estabilizando até os 50 anos e seu declínio passa a acontecer por volta dos 70 (Gomes et al, 2016).

Esse declínio de massa muscular leva a perda de força e resistência aeróbia no idoso, após os 65 anos acontece a perda de força de músculos das costas e antebraço, já a força das mãos diminui de forma rápida após os 40 anos (Oliveira et al, 2017).

No envelhecimento a força muscular é comprometida por conta também do enrijecimento dos tendões, o que interfere na prevenção as quedas, ocorrendo mais frequentemente torções e luxações por conta da perda da elasticidade dos tendões e ligamentos (Teixeira et al, 2019).

O declínio da força muscular afeta também os grupos musculares que auxiliam na respiração o que influencia diretamente na função pulmonar desses indivíduos, por conta da perda principalmente das fibras musculares do tipo II que são as de contração rápida (Fechine \& Trompieri, 2012).

Estudos indicam que $60 \%$ dos adultos sedentários terão declínio na fibra tipo II após os oitenta anos de idade, a atrofia dessa fibra se relaciona com a redução da força, entendendo que a perda de força e potência no envelhecimento está relacionada tanto com a quantidade quanto a qualidade das proteínas nas unidades contráteis do músculo (Gomes et al, 2016).

Para a maioria dos autores essa diminuição acontece por conta do envelhecimento da massa óssea e também por conta da redução da água corporal, lembrando que essas alterações na massa muscular, gordura e massa óssea estão diretamente relacionadas com o sedentarismo (Oliveira et al, 2017).

\subsection{Prevenção do declínio músculo-esquelético no envelhecimento}

Os níveis de hormônio do crescimento em declínio e a falta da prática de exercício físico também contribuem para $40 \%$ das perdas do tecido muscular, os idosos acabam por não fazer atividades físicas, apresentando assim uma maior porcentagem de gordura e um menor teor de massa muscular, quando comparados aos idosos que fazer atividades físicas habitualmente (Leitão et al, 2018).

O decréscimo da atividade física diminui os gastos de energia em repouso reduzindo o efeito térmico dos alimentos, isso está inteiramente ligado com o acúmulo de gordura em idosos (Teixeira et al, 2019).

Um dos principais sinais do envelhecimento é a menor capacidade para atividades laborais do trabalho, a adaptação ao ambiente e independência funcional, exercícios físicos melhoram a função muscular e diminui a frequência de quedas melhorando a qualidade de vida do indivíduo idoso (Aciole \& Batista, 2013). 
O exercício físico realizado de maneira continua durante a vida pode atuar com fator de prevenção as comorbidades da velhice, exercícios de resistência além de proporcionar aumento da massa muscular minimizam e até podem reverter à síndrome de fragilidade física na velhice (Leitão et al, 2018).

Idosos que ao longo da vida se mantém ativos apresentam diversos ganhos a saúde, melhorando sua capacidade muscular (Teixeira et al, 2019).

Os discos vertebrais dos idosos perdem a porção do conteúdo de água que é importantíssimo para absorver choques, o que torna mais fibrosa comprimindo os discos reduzindo a coluna vertebral o que leva a perda subsequente da altura (Aciole \& Batista, 2013).

Na terceira idade a queda é um problema de saúde pública, resultando no aumento de gastos públicos com a saúde e diminuição da qualidade de vida desses indivíduos, estudos demonstram que as quedas são mais comuns no sexo feminino acima e 80 anos (Leitão et al, 2018).

Esses dados entram em concordância com o estudo de Pimentel et al (2018) que associou as quedas com a qualidade de vida do idosos de uma comunidade, ao aplicar o questionário de qualidade de vida Short Form Health Survey-36 (SF-36), ele percebeu que os idosos que mais sofriam quedas tinham pior média no domínio de aspectos emocionais, porque eles adquiriam pensamentos negativos com medo de novas quedas.

O Medo de cair pode limitar atividades o dia a dia do idoso, ficando restrito ao leito, o que ajuda na síndrome de imobilidade (Pimentel et al, 2018). O Brasil vem avançando com o cuidado a saúde da terceira idade, com a promoção da saúde, prevenção das incapacidades funcionais e a integridade da atenção a esses idosos na estratégia de saúde da família (Leitão et al, 2018).

Até 1999 não existia nenhuma política que fosse específica para essa população, hoje em dia o direito a saúde no envelhecimento é largamente protegido no Brasil com várias diretrizes como, por exemplo, a lei Orgânica da Saúde $\left(\mathrm{n}^{\circ}\right.$ 8080/90), estatuto do Idoso - Instituído pela Lei 10741 de 1998 e a política Nacional de Saúde do Idoso (PNSI) - Instituída em 1999 (Aciole \& Batista, 2013).

\subsection{Papel da Fisioterapia no declínio músculo-esquelético no envelhecimento}

A Fisioterapia entra com um papel importantíssimo tanto na prevenção de quedas, como na reabilitação desses indivíduos, utilizando de técnicas como, alongamentos, fortalecimento muscular, treino de marcha e equilíbrio, melhora da propriocepção, buscando a manutenção ou melhoria da capacidade funcional, redução das incapacidades e limitações e proporcionando maior independência funcional (Oliveira et al, 2017).

A Fisioterapia inclui também orientações além da assistência para o desenvolvimento de atividades, o fisioterapeuta está mais que apto a avaliar, tratar e prevenir distúrbios cardiovasculares, respiratórios, neurológicos e musculoesqueléticos que possam interferir e provocar limitações à funcionalidade do organismo, com fins de promover a independência funcional, reduzindo o risco de quedas e contribuindo, assim, com a melhora da autoestima do idoso (Meres, 2018).

Ao contrário do que muitos pensam a fisioterapia preventiva é importante para o bem estar do idoso, pois a fisioterapia atua preservando as funções motoras com o objetivo de adiar e minimizar as possíveis patologias associadas ao envelhecimento (Teixeira et al, 2019).

Além de todo o tratamento fisioterapêutico ele também faz uma avaliação do ambiente que o idoso vive, para melhorar ou modificar possíveis riscos domésticos de quedas (Meres, 2018).

$\mathrm{O}$ tratamento fisioterapêutico contribui em todos os processos, desde o retardo de alterações fisiológicas, contribuindo para o aumento de força, melhora da flexibilidade e do equilíbrio, que são importantes para a independência funcional dos idosos, evitando assim o surgimento do medo de cair, pois esses indivíduos passam a reconhecer a própria independência e 
limitações (Rodrigues et al, 2016).

É comprovado cientificamente que a prática regular de atividades físicas, melhora a qualidade de vida no envelhecimento globalmente auxiliando na prevenção de quedas, oferecendo maior segurança na realização das atividades de vida diária, favorecendo o contato social, reduzindo o risco de doenças crônicas, melhorando a saúde física e mental e a performance funcional, autonomia e independência funcional (Meres, 2018).

Segundo Rodrigues; Barbeito; Junior, (2016) com os resultados do seu estudo, eles mencionam a importância de um programa de atividades físicas voltadas para prevenção de quedas na terceira idade, contendo exercícios de força, flexibilidade e equilíbrio, pois o processo de envelhecimento tem como consequência a sarcopenia, a diminuição da flexibilidade e mobilidade articular e maior instabilidade, ajudando também nas tarefas do cotidiano, como por exemplo, subir e descer escadas.

Lembrando que Rodrigues; Barbeito; Junior, (2016) informam que esses programas devem levar em conta diversos fatores, como sociais e deve-se buscar a inserção dessa população na sociedade estimulando sua interação social.

A cinesioterapia trabalhada na fisioterapia vem com o objetivo de aprimorar o equilíbrio estático e dinâmico nos idosos, esses exercícios apresentam eficácia, isso já é comprovado cientificamente reduzindo o número de quedas, além do aprimoramento físico entra também os efeitos na duração da morbidade que interfere nas relações sociais (Meres, 2018).

A cinesioterapia trabalha com exercícios passivos e ativos, a escolha dependerá da avaliação fisioterapêutica que deve ser feita minunciosamente, esses exercícios devem ser feitos regularmente e tem objetivo de estabilizar a postura, treinar marcha propiciando a melhora do equilíbrio e associar o fortalecimento da musculatura dos membros inferiores e superiores e tronco, flexões articulares, mudanças de decúbito, técnicas respiratórias e proprioceptivas (Teixeira et al, 2019).

Outra técnica da fisioterapia é a hidrocinesioterapia que também traz benefícios prevenindo quedas e fortalecendo a musculatura e a melhora do equilíbrio e como é feito dentro da água minimiza o risco de quedas (Meres, 2018).

Na hidrocinesioterapia existe a combinação da propriedade físicas da água com a cinesioterapia mencionada anteriormente, a água traz mais segurança para o paciente realizar o exercício (Leitão et al, 2018).

Sendo a hidrocinesioterapia atividade ideal para prevenir, tratar e retardar o surgimento das disfunções físicas decorrentes do envelhecimento, além da possibilidade de manter e melhorar a funcionalidade preservada do idoso (Vieira; et al, 2019).

É crucial para o fisioterapeuta trabalhar o equilíbrio, pois idosos com histórico de quedas apresentam uma marcha oscilante e relação aos que não tem esse histórico, para idosos que caem com muita frequência é difícil ficar em posição estática por muito tempo, o que pode estar ligado a novas quedas (Meres, 2018).

Em um estudo clínico, prospectivo, aleatório que Avelar; Bastone; Alcântara; Gomes (2010) se baseou no fortalecimento dos membros inferiores em uma amostra de 36 idosos, eles utilizaram exercícios tanto da cinesioterapia como da hidrocinesioterapia e apresentou melhoras no equilíbrio estático e dinâmico.

Em outro estudo randomizado de Roma et al. (2013) que foi feito com idosos sedentários, sem contraindicações para atividade física que foram distribuídos em dois grupos, o Grupo Resistido que realizou 6 exercícios por treino, 2 vezes por semana e o Grupo Aeróbio que realizou atividade por 30 minutos, 2 vezes por semana, eles obtiveram resultados positivos nos dois grupos, pois ambos apresentaram melhora na aptidão física.

Deve-se reforçar a importância de se fazer atividade física resistida e aeróbia na velhice, pois melhora a aptidão física e consequentemente a funcionalidade, aumentando a massa muscular reduzindo assim os riscos de queda, o fisioterapeuta tem o dever de incentivar os idosos a praticarem regularmente, mostrando os inúmeros benefícios para sua saúde (Roma et al, 2013).

No ensaio clínico randomizado, cego de Lustosa et al. (2011) fez em seu estudo um programa de resistência muscular 
na capacidade funcional e na força muscular dos extensores do joelho em idosas pré-frágeis e observou a melhora da potência muscular e do desempenho funcional, melhorando a independência e qualidade de vida dos idosos.

Já os resultados do estudo de Nascimento et al. (2012) evidenciaram melhora no desempenho funcional, com exercícios de dupla tarefa e cinesioterapia dos pacientes idosos com Alzheimer.

Afieri et al. (2010), em estudo clínico simples-cego e aleatorizado com braços paralelos, fez o uso de testes clínicos para verificação do controle postural em idosos saudáveis submetidos a programas de exercícios físicos e viu que a prática de exercícios físicos interfere positivamente no controle postural de idosos.

Em um ensaio clínico aleatório de Barboza et al. (2014) observou que a fisioterapia associada à dança proporcionou melhora significativa do equilíbrio, agilidade e flexibilidade em idosos.

Em outro estudo de Costa et al. (2012) que realizou ensaio clínico randomizado e avaliou a força de preensão manual em idosas com baixa massa óssea e um programa de exercícios, notou benefícios com melhora significativa na força de preensão manual em relação ao grupo controle.

Sá, Bachion e Menezes (2012) fizeram um estudo em que tiveram ganhos significantes para equilíbrio, força muscular de membros inferiores e amplitude de movimento de flexão dos ombros com a fisioterapia.

Em um ensaio clínico randomizado controlado de Aveiro, Driusso, Santos (2013), observaram a melhora da qualidade de vida e de alguns aspectos psicológicos influenciados pela prática de atividades físicas.

O Fisioterapeuta deve seguir também com orientações gerais a esses idosos, como, por exemplo, sobre as adaptações domiciliares que de acordo ao concelho Nacional de Educação (CNE), o fisioterapeuta é apto a atuar em todos os níveis da atenção à saúde em programas de promoção, proteção, prevenção e recuperação da saúde individual e coletiva (Meres, 2018).

Segundo Meres (2018) é essencial que o Fisioterapeuta, trabalhe em multidisciplinarmente em ações que estimulem saúde, como por exemplo, projetos de alimentação balanceada, atividades físicas frequentes e outros hábitos de vida saudáveis, além de orientar e intervir na organização do ambiente domiciliar a fim de reduzir os riscos de quedas.

Um ambiente ideal para essa população precisa de uma boa ventilação, temperatura agradável, iluminação adequada, minimização de ruídos do tráfego, de aparelhos e de outros sons que possam ser desagradáveis, iluminação deve ser difusa e indireta para assim atender às necessidades características da idade, a casa deve ser livre de obstáculos dentre outros (Aciole \& Batista, 2013).

Considerando a participação da fisioterapia na atenção primária, é importante focar no desenvolvimento de estratégias com o intuito de estimular hábitos de vida mais saudáveis nos idosos como a prática de atividade física, alimentação equilibrada, orientações domiciliares para melhorar a qualidade de vida dessa população (Oliveira et al, 2017).

\section{Conclusão}

Esse estudo concluiu que com o índice de envelhecimento crescente e o aumento das quedas na população idosa esse estudo se tornou de grande relevância, pois esse evento pode provocar graves consequências, diminuindo a autonomia, causando dependência funcional e diminuição na qualidade de vida.

A Fisioterapia entra como forte propiciadora da melhor qualidade de vida dessa população, com suas técnicas trata, mas também previne, melhora a saúde do idoso globalmente, melhorando sua capacidade funcional, fortalecimento da musculatura, melhorando seu equilíbrio estático e dinâmico, prevenindo futuras quedas.

Com esse trabalho se faz necessário à criação de mais estratégias para prevenção de quedas, diminuição dos fatores de risco e também para informar a essa população as alterações que sofrerão ao longo do tempo. 


\section{Referências}

Aciole, G. G., \& Batista, L. H. (2013). Promoção da saúde e prevenção de incapacidades funcionais dos idosos na estratégia de saúde da família: a contribuição da fisioterapia. Saúde em Debate, Rio de Janeiro, 37(96), 10-19.

Alfieri, F. M., Riberto, M., Gatz, L. S., Ribeiro, C. P. C., \& Battistella, L. R. (2010), Uso de testes clínicos para verificação do controle postural em idosos saudáveis submetidos a programas de exercícios físicos. acta fisiatra, 17(4), 153 - 158.

Araújo, A. P. S., Bertolini, S. M. M. G., \& Junior, J. M. (2014). Alterações morfofisiológicas decorrentes do processo de envelhecimento do sistema musculoesquelético e suas consequências para o organismo humano. Biológicas \& Saúde, 4(12).

Aveiro, M. C., Driusso, P., Santos, J. G., Kiyoto, V. D., \& Oishi, J. (2013). Efeitos de um programa de fisioterapia sobre a qualidade de vida de mulheres idosas da comunidade: estudo randomizado controlado. Fisioter. Mov, Curitiba, 26(3), 503-513.

Barboza, N. M., Floriano, E. N., Motter, B. L., Silva, F. C., \& Smaili Santos, S. M. S. (2014). Efetividade da fisioterapia associada à dança em idosos saudáveis: ensaio clínico aleatório. Rev. Bras. Geriatr. Gerontol. 17(1), 87-98.

Costa, et al. (2012). Efeitos de um programa de exercícios em grupo sobre a força de preensão manual em idosas com baixa massa óssea. Arq Bras Endocrinol Metab, 56(5).

Esquenazi, D., Silva, S. R. B., \& Guimarães, M. A. M. (2014). Aspectos fisiopatológicos do envelhecimento humano e quedas em idosos. Revista HUPE, 13(2), 11-20.

Fechine, B. R. A., \& Trompieri, N. (2012). O processo de envelhecimento: as principais alterações que acontecem com o idoso com o passar dos anos. Revista cientifica internacional, 20(1), 106-194.

Gomes, A. R. L. G., Campos, M. S., Mendes, M. R. P., \& Moussa, L. (2016). A influência da fisioterapia, com exercícios de equilíbrio, na prevenção de quedas em idosos. FisiSenectus. Unochapecó, 4(1), 4-11.

Leitão, S. M., Oliveira, S. C. O., Rolim, L. R., Carvalho, R. P., Filho, J. M. C., \& Junior, A. A. P. (2018). Epidemiologia das quedas entre idosos no Brasil: uma revisão integrativa de literatura. Geriatr Gerontol Aging, 12(3), 172-9.

Lustosa, L, P., Silva, J. P., Coelho, F. M., Pereira, D. S., Parentoni, A. N., \& Pereira, S. M. (2011). Efeito de um programa de resistência muscular na capacidade funcional e na força muscular dos extensores do joelho em idosas pré-frágeis da comunidade: ensaio clínico aleatorizado do tipo crossover. Rev. bras.fisioter, 15(4).

Nascimento, et al. (2012). Efeitos do exercício físico sobre distúrbios neuropsiquiátricos e atividades instrumentais da vida diária em mulheres com doença de Alzheimer: um ensaio clínico controlado. Rev. bras. Fisioter, 16(3).

Núbia, C. P. A., Alessandra C. B., Marcus A. A., \& Wellington F. G. (2010). Efetividade do treinamento de resistência à fadiga dos músculos dos membros inferiores dentro e fora d'água no equilíbrio estático e dinâmico de idosos. Rev Bras Fisioter, São Carlos, 14(3), 229-36.

Oliveira, H. M. L., Rodrigues, L. F., Caruso, M. F. B., \& Freire, N. S. A. (2017). Fisioterapia na prevenção de quedas em idosos: revisão de literatura. Revista Interdisciplinar de Estudos Experimentais, 9, 43-47.

Pimentel, W. R. T. et al. (2018). Quedas entre idosos brasileiros residentes em áreas urbanas: ELSI-Brasil. Rev Saude Publica. 52(2), 1-9.

Rodrigues, G. D., Barbeito, A. B., \& Junior, E. D. A. (2016). Prevenção de quedas no idoso: revisão da literatura brasileira. Revista Brasileira de Prescrição e Fisiologia do Exercício, São Paulo. 10(59), 431-437.

Roma, M. F. B., Busse, A. L. B., Betoni, R. A., Melo, A. C., Kong, J., Santarem, J. M., \& Filho, W. J. (2013). Efeitos das atividades físicas resistida e aeróbia em idosos em relação à aptidão física e à funcionalidade: ensaio clínico prospectivo. einstein. 11(2), 153-7.

Sá, A. C. A. M., Bachion, M. M., \& Menezes, R. L. (2012). Exercício físico para prevenção de quedas: ensaio clínico com idosos institucionalizados em Goiânia, Brasil. Ciênc. saúde coletiva, 17(8).

Teixeira, D. K. S., Andrade, L. M., Santos, J. L. P. S., \& Caires, E. S. (2019). Quedas em pessoas idosas: restrições do ambiente doméstico e perdas funcionais. Rev. Bras. Geriatr. Gerontol, 22(3), 1-10.

Vieira, R. A., D’ Alessandro, C. C., Reis, E. D. S., Paiva, J. P., Xavier, K. L., Rodrigues, L. A., Barbalho, M. C., Aquino, M. O., Maia, P. C., \& Ribeiro, V. M.L. (2019). A atuação da fisioterapia na prevenção de quedas em pacientes com osteoporose senil. Fisioterapia Brasil, 3(2). 